\title{
Risk Factors and Outcomes of Postoperative Neck Hematomas: An Analysis of 5,900 Thyroidectomies Performed at a Cancer Center
}

\author{
Andre Ywata de Carvalho ${ }^{1}$ Camila Couto Gomes ${ }^{10}$ Thiago Celestino Chulam ${ }^{10}$ \\ Jose Guilherme Vartanian ${ }^{10}$ Genival Barbosa Carvalho ${ }^{10}$ Renan Bezerra Lira ${ }^{10}$ \\ Hugo Fontan Kohler ${ }^{10}$ Luiz Paulo Kowalski ${ }^{10}$
}

${ }^{1}$ AC Camargo Cancer Center, São Paulo, SP, Brazil Int Arch Otorhinolaryngol 2021;25(3):e421-e427.
Address for correspondence Andre Ywata de Carvalho, MD, MBA, PhD, AC Camargo Cancer Center. Rua Professor Antonio Prudente, 211, 01509-010, Sao Paulo, Brazil (e-mail: andreywata@bol.com.br).

\begin{abstract}
Introduction Postoperative neck hematoma (PNH) is an uncommon but potentiallylethal complication of thyroid surgery.

Objective To identify the risk factors for postthyroidectomy hematoma requiring reoperation, the timing, the source of the bleeding, the related respiratory distress requiring tracheotomy, and the late outcomes.

Methods We retrospectively analyzed the records of 5,900 consecutive patients submitted to surgery for thyroid diseases at a single institution.

Results In total, PNH occurred in $62(1.1 \%)$ patients. Most cases of bleeding occurred within the first 6 hours after thyroidectomy, but $12.5 \%$ of the hematomas were observed after 24 hours. Obvious bleeding points were detected in $58.1 \%$ of the patients during the reoperation, with inferior thyroid artery branches and superior thyroid vessels being the most frequent bleeding sources. Only two patients required urgent tracheostomy. There were no hematoma-related deaths. Permanent hypoparathyroidism and recurrent laryngeal nerve injury are more frequent following reoperation for PNH. The factors significantly associated with PNH were: older age, concurrent lymph node dissection, and chronic lymphocytic thyroiditis. Gender, previous neck irradiation, presentation at diagnosis (symptomatic or incidental), substernal goiter, thyroidectomy for hyperthyroidism, prior thyroid surgery, malignant histology, the extent of the surgery (total versus non-total thyroidectomy), the use of energy-based

\section{Keywords}

- thyroid

- hematoma

- complication

- surgery

- reoperation vessel sealing devices, the use of the hemostatic agent Surgicel, and the placement of a surgical drain were not significantly associated with PNH.

Conclusion Hematoma after thyroid surgery is an uncommon complication, but it is related to significant postoperative morbidity. A better understanding of the risk factors and of the time until hematoma formation can help target high-risk patients for preventive measures and closer postoperative observation.
\end{abstract}

received

November 15, 2019

accepted

June 5, 2020

published online

September 30, 2020
DOI https://doi.org/ 10.1055/s-0040-1714129. ISSN 1809-9777. (c) 2020. Fundação Otorrinolaringologia. All rights reserved.

This is an open access article published by Thieme under the terms of the Creative Commons Attribution-NonDerivative-NonCommercial-License, permitting copying and reproduction so long as the original work is given appropriate credit. Contents may not be used for commercial purposes, or adapted, remixed, transformed or built upon. (https://creativecommons.org/ licenses/by-nc-nd/4.0/)

Thieme Revinter Publicações Ltda., Rua do Matoso 170, Rio de Janeiro, RJ, CEP 20270-135, Brazil 


\section{Introduction}

In recent years, the number of patients undergoing thyroid surgery has increased, largely due to an increase in the diagnosis of small thyroid cancers. ${ }^{1}$ Although thyroidectomy is a relatively safe procedure, it may be associated with some clinically-concerning postoperative complications. Postoperative neck hematoma ( $\mathrm{PNH}$ ) is an uncommon but potentially life-threatening complication of thyroid surgery. In severe cases, PNH leads to airway compression, causing acute respiratory distress or even death, and emergency surgical treatment is usually required. It is particularly relevant to short-stay thyroidectomy on an outpatient basis. ${ }^{2}$ According to Margolick et $\mathrm{al}^{3}{ }^{3}$ neck hematoma is the most common reason for reoperation, while postoperative hypocalcemia is the most frequent reason for hospital readmission.

A better understanding of the risk factors to develop postthyroidectomy hemorrhage could help surgeons minimize the risk of PNH and select patients suitable for short-stay thyroid surgery. In the present study, we aimed to identify the risk factors associated with neck hematoma requiring surgical reintervention after thyroidectomy. We also reviewed our experience with $\mathrm{PNH}$, analyzing the time course of events and late outcomes.

\section{Materials and Methods}

\section{Patients and Data Collection}

Data on patients undergoing thyroid surgery between January 1996 to December 2015 were retrospectively extracted from medical records. We excluded patients with concurrent head and neck cancers. We analyzed the patients and the operative data, as well the postoperative outcomes.

\section{Variables Analyzed}

The following patient characteristics were assessed: age, gender, previous neck irradiation, presentation at diagnosis (symptomatic or incidental), substernal goiter, hyperthyroidism, previous thyroid surgery, pathological findings (malignant versus benign) and pathological lymphocytic thyroiditis. The operative characteristics analyzed were: extent of the surgery (total versus non-total thyroidectomy), concurrent lymph-node neck dissection, the use of energy-based vessel sealing devices - harmonic scalpel (Ethicon, Inc., Bridgewater, NJ, US) or LigaSure (Medtronic, Dublin, Ireland) - versus the conventional technique (electrocoagulation and "clamp-and-tie"), the use of the hemostatic agent Surgicel (Ethicon, Inc.), and the placement of a surgical drain. The time until the development of a $\mathrm{PNH}$ was determined by the interval in hours from the end of the thyroidectomy until the start of the reintervention operation. Given the difficulty in documenting when a wound hematoma is first identified, we think the time interval from the initial operation until the reoperation is a more reliable measure. The source of the bleeding, when identified, was noted, as well as the need for tracheostomy to ensure airway patency.

\section{Outcomes}

Information about hematoma-related deaths and the need for urgent tracheostomy were recorded, as well as the postoperative complications. Hypoparathyroidism was defined by altered laboratory tests and/or the presence of symptoms of hypocalcemia postoperatively, and it was classified as transient or permanent if the duration was longer than 6 months. Likewise, the rates of recurrent laryngeal-nerve dysfunction (temporary or permanent) were compared between patients that had and those who did not have PNH. Wound infections were defined by the need for antibiotic therapy or the presence of purulent content during drainage.

\section{Statistical Analysis}

All statistical analyses were performed using the Stata software (StataCorp., College Station, TX, US), version 10. For the continuous variables, the results are reported as median and range values, if not stated otherwise. Frequency distribution was used to describe the categorical variables (number of cases and relative percentage). For the statistical evaluation of the differences between the groups, the nonparametric Mann-Whitney U test was applied. The categorical variables were analyzed using the Chi-squared test for $2 \times 2$ tables, and the Fisher exact test was used when at least 1 expected frequency was lower than 5 . To verify the normality of the data, the Shapiro-Wilk test was applied. Odds ratios (ORs) and their respective $95 \%$ confidence intervals (95\%Cls) were estimated based on a logistic regression model and the stepwise forward technique, and a significance level of $10 \%$ was adopted to select the variables.

\section{Results}

\section{Demographics}

A total of 5,900 patients who underwent thyroid surgery were included. The median age was 45.3 years (range: 7-96 years), and 4,826 patients ( $82 \%$ ) were women. Most patients - 4,554 (77.3\%) - were operated because of nonpalpable nodules incidentally noted during a radiologic procedure. Clinically, 148 patients (2.5\%) had substernal goiter. Only 180 patients $(3.2 \%)$ had been submitted surgery due to hyperthyroidism. In total, 264 patients (4.9\%) had previously undergone thyroid surgery. History of previous neck (thyroid) irradiation was observed in 103 cases (1.7\%).

\section{Surgery \\ Most patients (91\%) underwent total thyroidectomy. Con- current lymph-node dissection was performed in 717 cases (12.2\%): 547 patients (76.3\%) had level-VI neck clearance, and 170 (23.7\%) underwent central and lateral neck dissection. Energy-based surgical sealing devices were used in 1,505 patients (25.5\%): the harmonic scalpel, in 1,072 (18.2\%), and LigaSure, in 433 patients (7.3\%). In the other 4,395 patients (74.5\%), the conventional hemostasis technique (coagulation with electrocautery and ligation of blood vessels) was used. An adjunctive hemostatic agent (Surgicel) and a surgical drain were used in 1,631 (27.6\%) and 2,327 patients (41.8\%) respectively.}




\section{Histology}

The final pathology reports showed malignancy in 3,928 patients (66.6\%), and concomitant lymphocytic thyroiditis in 1,855 patients (31.4\%).

\section{Postthyroidectomy Hematoma}

Out of 5,900 patients included in the study, 62 (1.1\%) presented wound hematoma requiring reintervention. Out of these 62 patients, 56 (90.3\%) had available information on the time of the onset of the postoperative bleeding. Out of these patients, 35 (62.5\%) had wound hematoma within 6 hours postthyroidectomy, while $12(21.4 \%)$ had wound hematoma between 6 and 12 hours postoperatively, 2 (3.6\%), between 12 and 24 hours, and 7 (12.5\%) more than 24 hours postthyroidectomy (-Fig. 1). During the study period, only two patients (3.2\%) underwent tracheotomy related to airway compression. Obvious bleeding points were detected in 36 patients (58.1\%) during the reoperation. Inferior thyroid artery branches, superior thyroid vessels, the Berry ligament, the anterior jugular vein, and the surface of the strap muscles were the most frequent bleeding sources (-Table 1).

\section{Risk Factors for Postthyroidectomy Hematoma}

- Table 2 presents the bivariate analysis comparing the patients who did and did not present cervical hematoma.
Table 1 Source of the postthyroidectomy bleeding

\begin{tabular}{|l|l|}
\hline No obvious source & $26(41.9 \%)$ \\
\hline Inferior thyroid artery branch & $10(16.1 \%)$ \\
\hline Superior thyroid vessel & $8(12.9 \%)$ \\
\hline Berry ligament & $5(8.1 \%)$ \\
\hline Anterior jugular vein & $4(6.5 \%)$ \\
\hline Strap muscle & $4(6.5 \%)$ \\
\hline Thyroid remnant after lobectomy & $2(3.2 \%)$ \\
\hline Middle thyroid vein & $1(1.6 \%)$ \\
\hline External jugular vein & $1(1.6 \%)$ \\
\hline Other sources of arterial bleeding & $1(1.6 \%)$ \\
\hline
\end{tabular}

Higher incidence proportions of PNH were significantly associated with older age, concurrent lymph-node neck dissection and chronic lymphocytic thyroiditis (- Table 3 ). Gender, previous neck irradiation, symptoms at presentation (non-incidental diagnosis), substernal goiter, hyperthyroidism, previous thyroid surgery, malignant histology, the extent of the surgery, the use of energy-based vessel sealing devices, the use of a hemostatic agent, and the placement of a surgical drain were not associated with the occurrence of $\mathrm{PNH}$.

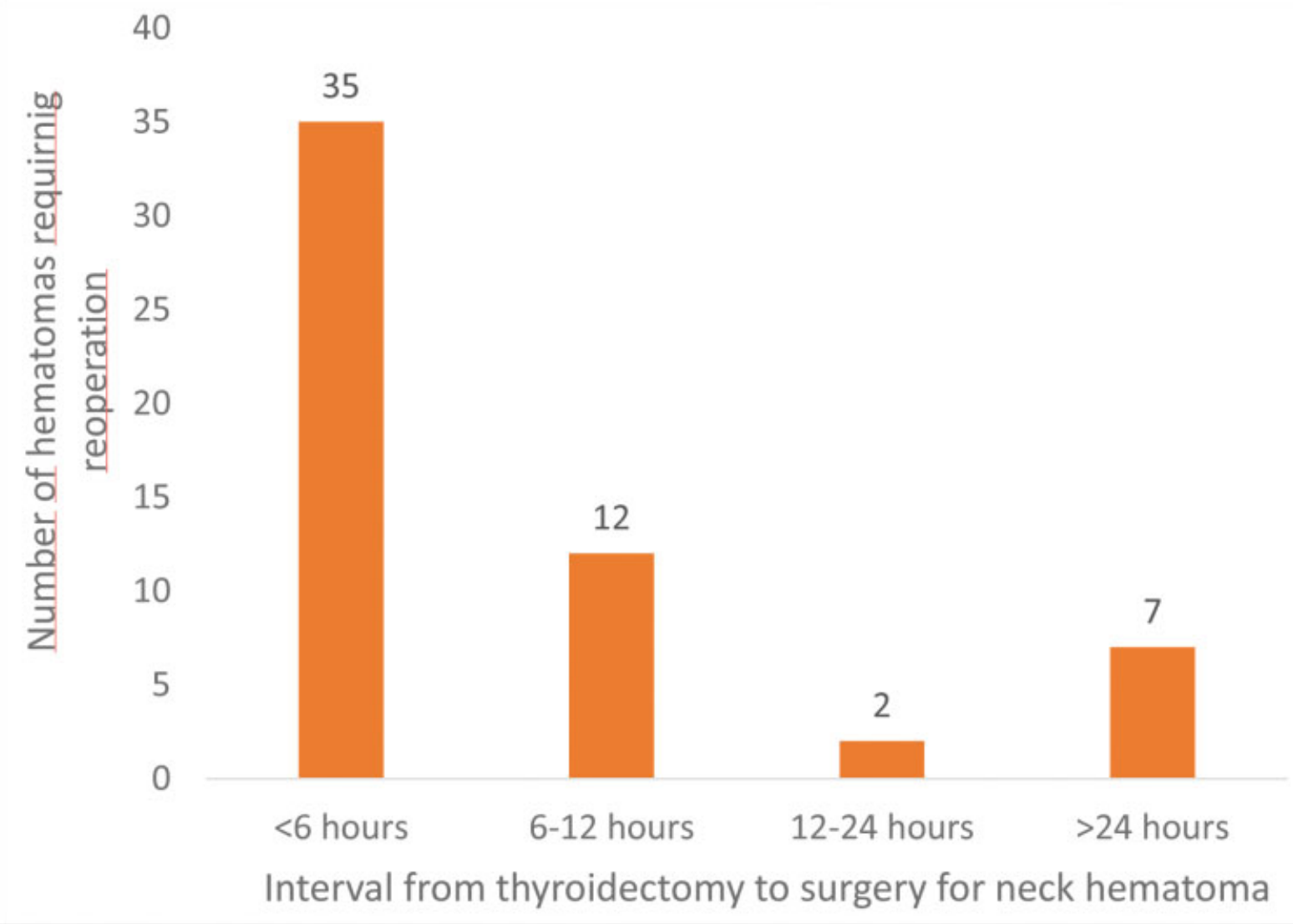

Fig. 1 Interval from thyroidectomy to surgery for neck hematoma. 
Table 2 Patient characteristics and postoperative neck hematoma

\begin{tabular}{|c|c|c|c|c|c|}
\hline \multicolumn{2}{|l|}{ Characteristics } & Patients, N (\%) & No hematoma & Hematoma & $p$-value \\
\hline \multicolumn{2}{|l|}{ Total } & $5,900(100)$ & $5,838(89.9)$ & $62(1.1)$ & \\
\hline \multirow[t]{3}{*}{ Age (years) } & Mean (range) & $45.3(7-96)$ & $45.3(7-96)$ & $51.2(14-82)$ & $0.001^{* * *}$ \\
\hline & $<45$ years & $2,877(48.8)$ & $2,856(99.3)$ & $21(0.7)$ & \multirow[t]{2}{*}{$0.018^{*}$} \\
\hline & $\geq 45$ years & $3,023(51.2)$ & $2,982(98.6)$ & $41(1.4)$ & \\
\hline \multirow[t]{2}{*}{ Gender } & Female & $4,826(81.8)$ & $4,778(99.0)$ & $48(1.0)$ & \multirow[t]{2}{*}{$0.369^{*}$} \\
\hline & Male & $1,074(18.2)$ & $1,060(98.7)$ & $14(1.3)$ & \\
\hline \multicolumn{2}{|l|}{ Previous neck irradiation } & $103(1.7)$ & $103(100)$ & 0 & $0.627^{* *}$ \\
\hline \multicolumn{2}{|l|}{ Non-incidental diagnosis } & $1,346(22.7)$ & $1,327(98.6)$ & $19(1.4)$ & $0.120^{*}$ \\
\hline \multicolumn{2}{|l|}{ Substernal goiter } & $148(2.5)$ & $146(98.6)$ & $2(1.4)$ & $0.666^{* *}$ \\
\hline \multicolumn{2}{|l|}{ Hyperthyroidism } & $180(3.2)$ & $177(98.3)$ & $3(1.7)$ & $0.350^{*}$ \\
\hline \multicolumn{2}{|l|}{ Previous thyroid surgery } & $264(4.9)$ & $260(98.5)$ & $4(1.5)$ & $0.363^{* *}$ \\
\hline \multicolumn{2}{|l|}{ Malignant histology } & $3,928(66.6)$ & 3,885 (98.9) & $43(1.1)$ & $0.641^{*}$ \\
\hline \multicolumn{2}{|l|}{ Lymphocytic thyroiditis } & $1,855(31.4)$ & $1,827(98.5)$ & $28(1.5)$ & $0.019^{*}$ \\
\hline \multirow[t]{2}{*}{ Surgery extent } & Total thyroidectomy & $5,367(91)$ & $5,310(98.9)$ & $57(1.1)$ & \multirow[t]{2}{*}{$0.789^{*}$} \\
\hline & Non-total thyroidectomy & $533(9)$ & $528(99.1)$ & $5(0.9)$ & \\
\hline \multicolumn{2}{|c|}{ Concurrent neck dissection } & $717(12.2)$ & $704(98.2)$ & $13(1.8)$ & $0.033^{*}$ \\
\hline \multirow[t]{3}{*}{ Hemostasis technique } & Harmonic scalpel & $1,072(18.2)$ & $1,062(99.1)$ & $10(0.9)$ & \multirow[t]{3}{*}{ Not accessible } \\
\hline & Ligasure & $433(7.3)$ & $430(99.3)$ & $3(0.7)$ & \\
\hline & Conventional & 4,395 (74.5) & 4,346 (98.9) & $49(1.1)$ & \\
\hline \multicolumn{2}{|c|}{ Use of hemostatic agent (Surgicel) } & $1,631(27.6)$ & $1,621(99.4)$ & $10(0.6)$ & $0.042^{*}$ \\
\hline \multicolumn{2}{|c|}{ Placement of surgical drain } & $2,327(41.8)$ & $2,297(98.7)$ & $30(1.3)$ & $0.095^{*}$ \\
\hline
\end{tabular}

Notes:

${ }^{*}$ Chi-squared test.

"*Fisher exact test.

${ }^{* * *}$ Mann-Whitney U-test.

\section{Operative Outcomes}

No patients died during hospitalization due to cervical hematoma. The operative outcomes were summarized in -Table 4.

\section{Discussion}

Using an inpatient database of a single tertiary center, we investigated the incidence and risk factors for $\mathrm{PNH}$ requiring surgical reintervention in patients undergoing thyroidectomy. Among 5,900 eligible patients, only 62 (1.1\%) had neck hematoma. To our knowledge, this is the first study analyzing the factors influencing postthyroidectomy wound hematoma including a large sample from a cancer center in Brazil. The incidence of postoperative neck hematoma (1.1\%) was similar to that reported in the literature $(0.7 \%$ to $4.7 \%){ }^{4-9}$

In the present study, all patients were hospitalized for at least one day, and they were observed postoperatively for a minimum of seven days. We believe that this period of observation is long enough to capture a delayed occurrence of wound hematoma. We found that most 35 patients (62.5\%) had PNH during the same hospitalization within 6 hours of

Table 3 Multivariate analysis of risk factors for postoperative neck hematoma

\begin{tabular}{|l|l|l|l|l|}
\hline & Crude analysis & & Multivariate analysis \\
\hline Covariates & OR $(95 \% \mathrm{Cl})$ & $p$-value & OR $(95 \% \mathrm{Cl})$ & $p$-value \\
\hline Age $\geq 45$ years & $1.87(1.10-3.17)$ & 0.02 & $2.03(1.19-3.46)$ & 0.009 \\
\hline Lymphocytic thyroiditis & $1.81(1.09-2.99)$ & 0.021 & $1.78(1.07-2.95)$ & 0.026 \\
\hline Concurrent neck dissection & $1.93(1.04-3.58)$ & 0.036 & $2.03(1.09-3.8)$ & 0.026 \\
\hline Use of hemostatic agent & $2.00(1.01-3.94)$ & 0.046 & & \\
\hline Placement of surgical drain & $3.22(1.26-8.21)$ & 0.098 & & \\
\hline
\end{tabular}

Abbreviations: $95 \% \mathrm{Cl}$, 95\% confidence interval; OR, odds ratio. 
Table 4 Operative outcomes

\begin{tabular}{|l|l|l|l|}
\hline & $\begin{array}{l}\text { No } \\
\text { hematoma }\end{array}$ & Hematoma & $p$-value \\
\hline $\mathrm{N}$ & 5,838 & 62 & \\
\hline $\begin{array}{l}\text { Transient } \\
\text { hypocalcemia }\end{array}$ & $\begin{array}{l}1,795 \\
(30.8 \%)\end{array}$ & $27(43.6 \%)$ & $0.031^{*}$ \\
\hline $\begin{array}{l}\text { Permanent } \\
\text { hypoparathyroidism }\end{array}$ & $304(5.4 \%)$ & $7(12.1 \%)$ & $0.037^{* *}$ \\
\hline $\begin{array}{l}\text { Transient recurrent } \\
\text { laryngeal-nerve palsy }\end{array}$ & $409(7 \%)$ & $7(11.3 \%)$ & $0.205^{* *}$ \\
\hline $\begin{array}{l}\text { Permanent recurrent } \\
\text { laryngeal-nerve injury }\end{array}$ & $139(2.4 \%)$ & $5(8.1 \%)$ & $0.017^{* *}$ \\
\hline Postoperative infection & $100(1.7 \%)$ & $3(4.8 \%)$ & $0.094^{* *}$ \\
\hline
\end{tabular}

Notes:

*Chi-squared test.

"Fisher exact test.

the thyroidectomy, which is often reported in the literature. ${ }^{5,8,10}$ Godballe et $\mathrm{al}^{8}$ reported that the median time for the onset of postoperative hemorrhage was 3 hours (range 0105 hours $).{ }^{8}$ In a multi-institutional international case-control study including 207 patients, Campbell et al $^{11}$ reported that $47 \%$ of hematoma patients returned to the operation room within 6 hours, and 79\%, within 24 hours after thyroidectomy. ${ }^{11}$ But some authors ${ }^{12}$ reported longer periods of time until the onset of bleeding. Suzuki et al, ${ }^{13}$ analyzing 51,968 patients from a Japanese database, found a $\mathrm{PNH}$ rate of $1.8 \%$, with nearly $20 \%$ of wound hematomas occurring 3 or more days after the thyroidectomy. ${ }^{13}$ In the present series, 7 patients (12.5\%) had neck hematomas 24 hours postthyroidectomy. This relevant information when considering outpatient procedures.

The treatment for PNH depends on the symptomatology. Most hematomas need to be evacuated to prevent respiratory distress. When a severe hematoma with rapid mucosal edema and upper-airway swelling is detected, intubation or even tracheotomy are generally the first-step emergency treatments, rather than neck surgery. In the present study, we showed that only 2 (3.2\%) out of 62 patients with wound hematoma underwent tracheotomy before reoperation, and both cases were diagnosed before 6 hours postthyroidectomy. Although uncommon, postthyroidectomy bleeding can be lethal in $0.01 \%$ to $1.34 \%$ of patients. ${ }^{6,14}$ In the present study, no patients died due to cervical hematoma. This is largely due to early reoperation and fiber-optic-assisted tracheal intubation at the anesthetic induction.

The source of bleeding is identified during reoperation in $57 \%$ to $73.6 \%$ of patients. ${ }^{4,10}$ In the present review, we were able to find a source of bleeding in $58.1 \%$ of the cases. Inferior thyroid artery branches and superior thyroid vessels were the most frequent bleeding sources.

The rate of postoperative complications is often higher after reoperation for neck hematoma. ${ }^{12}$ We found higher rates of permanent hypoparathyroidism (12.1\% versus 5.4\%; $p=0.037)$ and permanent recurrent laryngeal nerve disfunction $(8.1 \%$ versus $2.4 \% ; p=0.017$ ) in patients undergoing cervical reoperation due to hematoma compared with patients without bleeding respectively. The rate of wound infection rate was also higher, but did not reach significance (4.8\% versus $1.7 \% ; p=0.094$ ).

Several risk factors for postthyroidectomy hematoma have been described in the literature. Consistent with findings from previous studies, we found that older age, ${ }^{6-9,13-16}$ inflammatory thyroid disease, ${ }^{6}$ and concurrent lymph-node neck dissection $^{13,15,16}$ were independent risk factors for neck hematoma. We also found a higher risk of developing: wound hematoma among male patients, ${ }^{6-10,13-17}$ substernal or huge goiters, ${ }^{7,18}$ and symptoms at presentation (non-incidental diagnosis), but they did not reach statistical significance.

Previous studies revealed that thyroid histology, be it benign ${ }^{11,16}$ or malignant, ${ }^{8}$ can impact the postsurgical bleeding. Our findings are consistent with recent meta-analyses that failed to show any relationship between the pathological findings and the risk of hematoma formation. ${ }^{15,16,19}$

There is no consensus on the impact of the extent of the thyroid resection on postoperative bleeding. A higher risk of developing a postthyroidectomy hematoma has been described both after total ${ }^{13-16}$ and partial ${ }^{6}$ resections. We failed to show any association between the extent of the resection and the wound hematoma, possibly due to the small number of patients undergoing non-total thyroidectomies (9\%) in our series. Similarly, we did not find an increased risk of developing hematoma among patients with previous thyroid surgery, which was suggested in previous publications. ${ }^{5,10,14-16,20}$ Again, the present study included only a small number of patients with previous thyroid surgery (4.5\%), and most of these cases were completion thyroidectomies, which can be performed without significant increased risk because the second surgery would avoid the first operative field. ${ }^{21}$

Unlike the results in previous publications, ${ }^{7,11,13,15,16}$ the present study showed that patients with hyperthyroidism were not at a higher risk of developing wound hematoma. We speculate that a significant percentage of patients with toxic nodular goiter as a cause of hyperthyroidism in our series may influence the risk of postoperative hematoma. According to Quimby et al, ${ }^{19}$ Graves disease but not toxic nodular goiter significantly increases the risk of developing $\mathrm{PNH}$.

Energy-based surgical devices, such as bipolar scalpels and ultrasonic shears, are increasingly being used in thyroid surgery, aiming to improve surgical hemostasis. ${ }^{22,23}$ In fact, data comparing different options for the intraoperative ligation of blood vessels show significantly reduction in operative time, ${ }^{24,25}$ in blood loss, ${ }^{24,26}$ and shorter hospital length of stay $^{24,26}$ in favor of the harmonic scalpel or LigaSure over standard ligation and coagulation, with no difference in the rate of postoperative complications. ${ }^{24-27}$ The present study revealed no association between the use of energy-based vessel sealing devices and the occurrence of PNH. On the other hand, the use of a hemostatic agent (Surgicel) reduced the risk of PNH in the univariate analysis, but not in the multivariate analysis.

Surgicel, an oxidized cellulose mesh that adheres to tissue in areas of bleeding and helps form a hemostatic clot, is often used as an adjunctive hemostatic agent during thyroidectomy. ${ }^{11}$ However, Amit et $\mathrm{al}^{28}$ in a prospective randomized controlled 
trial, found no advantage in the application of Surgicel over conventional hemostasis in thyroid surgery. ${ }^{28}$ Actually, Surgicel has shown increases in drain output, time until drain removal, and hospital length of stay.

The use of a postoperative drain plays no role in most thyroid surgeries because it can increase pain and the hospital stay, with no difference in hematoma formation. ${ }^{29,30}$

In Brazil, thyroidectomy is widely performed by head and neck surgeons and otolaryngologists, but general surgeons also perform thyroid surgeries, mostly in nonacademic hospitals. It is known that thyroidectomies performed at high-volume hospitals ( $\geq 75$ thyroidectomies per year) have a lower propensity of resulting in $\mathrm{PNH}$, as compared with procedures performed in low-volume hospitals. ${ }^{15}$

We emphasize some important measures in the prevention of postthyroidectomy hematomas. Gentle tissue handling is strongly advised during thyroid surgery. Moreover, when using energy-based surgical devices, excessive traction should be avoided, as it may lead to premature rupture of blood vessels prior to proper sealing. Performing a Valsalva maneuver can help identify potential sources of bleeding before closing, and the performance of a loose reapproximation of the strap muscles could enable the earlier recognition of the hematoma and delay the lymphatic outflow blockage that leads to laryngeal edema. ${ }^{31}$ Controlling postoperative vomiting and hypertension is equally important.

Some limitations are noteworthy in the present study. Firstly, the present was a single-center retrospective cohort study without randomization of treatment assignment. Secondly, the procedures were performed by different surgeons with variables levels of expertise, which can influence the outcomes. ${ }^{14,32}$ Finaly, the size of the tumor and of the thyroid gland, ${ }^{18}$ the comorbidities (such as hypertension, chronic kidney disease and obesity), ${ }^{6}$ and use of anticoagulation medications, ${ }^{13}$ which were not available in the database, might act as confounding factors.

\section{Conclusion}

Hematoma after thyroid surgery is an uncommon complication, but it is related to significant postoperative morbidity. Some patients present neck hematoma 24 hours postthyroidectomy. Older age, thyroiditis and concurrent lymph-node neck dissection are independent risk factors for postoperative bleeding. Careful hemostasis during surgery is mandatory to prevent postoperative hematoma. Closer postoperative monitoring of the neck is recommended for patients with one or more risk factors 24 hours postthyroidectomy.

\section{Conflict of Interests}

The authors have no conflict of interests to declare.

\section{References}

1 Sun GH, DeMonner S, Davis MM. Epidemiological and economic trends in inpatient and outpatient thyroidectomy in the United States, 1996-2006. Thyroid 2013;23(06):727-733
2 Sørensen KR, Klug TE. Routine outpatient thyroid surgery cannot be recommended. Dan Med J 2015;62(02):A5016

3 Margolick J, Chen W, Wiseman SM. Systematic Review and MetaAnalysis of Unplanned Reoperations, Emergency Department Visits and Hospital Readmission After Thyroidectomy. Thyroid 2018;28(05):624-638

4 Christakis IA, Potylchansky E, Silva AM, et al. Cervical hematoma following an endocrine surgical procedure: The MD Anderson experience. Surgery 2016;160(02):377-383

5 Lang BH, Yih PC, Lo CY. A review of risk factors and timing for postoperative hematoma after thyroidectomy: is outpatient thyroidectomy really safe? World J Surg 2012;36(10):2497-2502

6 Weiss A, Lee KC, Brumund KT, Chang DC, Bouvet M. Risk factors for hematoma after thyroidectomy: results from the nationwide inpatient sample. Surgery 2014;156(02):399-404

7 Dehal A, Abbas A, Hussain F, Johna S. Risk factors for neck hematoma after thyroid or parathyroid surgery: ten-year analysis of the nationwide inpatient sample database. Perm J 2015;19(01):22-28

8 Godballe C, Madsen AR, Pedersen HB, et al. Post-thyroidectomy hemorrhage: a national study of patients treated at the Danish departments of ENT Head and Neck Surgery. Eur Arch Otorhinolaryngol 2009;266(12):1945-1952

9 Bergenfelz A, Jansson S, Kristoffersson A, et al. Complications to thyroid surgery: results as reported in a database from a multicenter audit comprising 3,660 patients. Langenbecks Arch Surg 2008;393(05):667-673

10 Liu J, Li Z, Liu S, Wang X, Xu Z, Tang P. Risk factors for and occurrence of postoperative cervical hematoma after thyroid surgery: A single-institution study based on 5156 cases from the past 2 years. Head Neck 2016;38(02):216-219

11 Campbell MJ, McCoy KL, Shen WT, et al. A multi-institutional international study of risk factors for hematoma after thyroidectomy. Surgery 2013;154(06):1283-1289, discussion 1289-1291

12 Burkey SH, van Heerden JA, Thompson GB, Grant CS, Schleck CD, Farley DR. Reexploration for symptomatic hematomas after cervical exploration. Surgery 2001;130(06):914-920

13 Suzuki S, Yasunaga H, Matsui H, Fushimi K, Saito Y, Yamasoba T. Factors Associated With Neck Hematoma After Thyroidectomy: A Retrospective Analysis Using a Japanese Inpatient Database. Medicine (Baltimore) 2016;95(07):e2812

14 Promberger R, Ott J, Kober F, et al. Risk factors for postoperative bleeding after thyroid surgery. Br J Surg 2012;99(03):373-379

15 Fan C, Zhou X, Su G, et al. Risk factors for neck hematoma requiring surgical re-intervention after thyroidectomy: a systematic review and meta-analysis. BMC Surg 2019;19(01):98

16 Liu J, Sun W, Dong W, et al. Risk factors for post-thyroidectomy haemorrhage: a meta-analysis. Eur J Endocrinol 2017;176(05): 591-602

17 Calò PG, Pisano G, Piga G, et al. Postoperative hematomas after thyroid surgery. Incidence and risk factors in our experience. Ann Ital Chir 2010;81(05):343-347

18 Agarwal A, Agarwal S, Tewari P, et al. Clinicopathological profile, airway management, and outcome in huge multinodular goiters: an institutional experience from an endemic goiter region. World J Surg 2012;36(04):755-760

19 Quimby AE, Wells ST, Hearn M, Javidnia H, Johnson-Obaseki S. Is there a group of patients at greater risk for hematoma following thyroidectomy? A systematic review and meta-analysis. Laryngoscope 2017;127(06):1483-1490

20 Hardman JC, Smith JA, Nankivell P, Sharma N, Watkinson JC. Reoperative thyroid surgery: a 20 -year prospective cohort study at a tertiary referral centre. Eur Arch Otorhinolaryngol 2015;272(06): 1503-1508

21 Li YJ, Wang YZ, Yi ZB, Chen LL, Zhou XD. Comparison of Completion Thyroidectomy and Primary Total Surgery for Differentiated Thyroid Cancer: A Meta-Analysis. Oncol Res Treat 2015;38(10): 528-531 
22 Garas G, Okabayashi K, Ashrafian H, et al. Which hemostatic device in thyroid surgery? A network meta-analysis of surgical technologies. Thyroid 2013;23(09):1138-1150

23 Siu JM, McCarty JC, Gadkaree S, et al. Association of Vessel-Sealant Devices vs Conventional Hemostasis With Postoperative Neck Hematoma After Thyroid Operations. JAMA Surg 2019;154(11): e193146

24 Ecker T, Carvalho AL, Choe JH, Walosek G, Preuss KJ. Hemostasis in thyroid surgery: harmonic scalpel versus other techniques-a meta-analysis. Otolaryngol Head Neck Surg 2010;143(01):17-25

25 Yao HS, Wang Q, Wang WJ, Ruan CP. Prospective clinical trials of thyroidectomy with LigaSure vs conventional vessel ligation: a systematic review and meta-analysis. Arch Surg 2009;144(12): 1167-1174

26 Pons Y, Gauthier J, Ukkola-Pons E, et al. Comparison of LigaSure vessel sealing system, harmonic scalpel, and conventional hemostasis in total thyroidectomy. Otolaryngol Head Neck Surg 2009; 141(04):496-501
27 Hua N, Quimby AE, Johnson-Obaseki S. Comparing Hematoma Incidence between Hemostatic Devices in Total Thyroidectomy: A Systematic Review and Meta-analysis. Otolaryngol Head Neck Surg 2019;161(05):770-778

28 Amit M, Binenbaum Y, Cohen JT, Gil Z. Effectiveness of an oxidized cellulose patch hemostatic agent in thyroid surgery: a prospective, randomized, controlled study. J Am Coll Surg 2013;217(02): 221-225

29 Sanabria A, Carvalho AL, Silver CE, et al. Routine drainage after thyroid surgery-a meta-analysis.J Surg Oncol 2007;96(03):273-280

30 Woods RS, Woods JF, Duignan ES, Timon C. Systematic review and meta-analysis of wound drains after thyroid surgery. Br J Surg 2014;101(05):446-456

31 Smith RB, Coughlin A. Thyroidectomy Hemostasis. Otolaryngol Clin North Am 2016;49(03):727-748

32 Kandil E, Noureldine SI, Abbas A, Tufano RP. The impact of surgical volume on patient outcomes following thyroid surgery. Surgery 2013;154(06):1346-1352, discussion 1352-1353 